\title{
Evaluation of Histological Changes in Back Muscle Injuries in Rats over Time
}

\author{
Koki Abe ${ }^{1}$, Kazuhide Inage ${ }^{1}$, Yoshihiro Sakuma ${ }^{2}$, Sumihisa Orita ${ }^{1}$, Kazuyo Yamauchi ${ }^{1}$, \\ Miyako Suzuki ${ }^{1}$, Go Kubota ${ }^{1}$, Yasuhiro Oikawa ${ }^{3}$, Takeshi Sainoh ${ }^{1}$, Jun Sato ${ }^{1}$, Kazuki Fujimoto ${ }^{1}$, \\ Yasuhiro Shiga ${ }^{1}$, Hirohito Kanamoto ${ }^{1}$, Kazuhisa Takahashi ${ }^{1}$, Seiji Ohtori ${ }^{1}$ \\ ${ }^{1}$ Department of Orthopaedic Surgery, Graduate School of Medicine, Chiba University, Chiba, Japan \\ ${ }^{2}$ Department of Orthopaedic Surgery, National Hospital Organization, Chiba Medical Center, Chiba, Japan \\ ${ }^{3}$ Department of Orthopaedic Surgery, Chiba Children's Hospital, Chiba, Japan
}

\section{Study Design: Animal model study.}

Purpose: The purpose of this study was to evaluate the histological variation in the injured muscle and production of calcitonin generelated peptide in rats over time.

Overview of Literature: Vertebral surgery has been reported to cause atrophy of the back muscles, which may result in pain. However, few reports have described the time series histological variation in the injured muscle and changes in the dominant nerve.

Methods: We used 30 male, 8-week-old Sprague-Dawley rats. The right and left sides of the paravertebral muscle were considered as the injured and uninjured sides, respectively. A $115 \mathrm{~g}$ weight was dropped from a height of $1 \mathrm{~m}$ on the right paravertebral muscle. Hematoxylin and eosin (H\&E) staining of the muscle was performed 1-3 weeks after injury for histological evaluation. Fluoro-Gold (FG) was injected into the paravertebral muscle. The L2 dorsal root ganglia on both sides were resected 1, 2, and 3 weeks after injury, and immunohistochemical staining for calcitonin gene-related peptide was performed.

Results: H\&E staining of the paravertebral muscle showed infiltration of inflammatory cells and the presence of granulation tissue in the injured part on the ipsilateral side 1 week after injury. Muscle atrophy occurred 3 weeks after injury, but was repaired via spontaneous replacement of muscle cells/fibers. In contrast, compared with the uninjured side, the percentage of cells double-labeled with FG and calcitonin gene-related peptide in FG-positive cells in the dorsal root ganglia of the injured side was significantly increased at each time point throughout the study period.

Conclusions: These results suggest that sensitization of the dominant nerve in the dorsal root ganglia, which may be caused by cicatrix formation, can protract injured muscle pain. This information may be helpful in elucidating the underlying mechanism of persistent pain after back muscle injury.

Keywords: Back muscles; Spine; Rats; Calcitonin gene-related peptide; Ganglia, sensory

\section{Introduction}

The incidence of spine surgery is estimated to be $1,800-$
2,050 and 400-725 million person-years in the United States and Japan, respectively [1]. According to research based on Medicare in the United States, the average rate

\footnotetext{
Received Apr 24, 2016; Revised Jun 20, 2016; Accepted Jun 21, 2016

Corresponding author: Koki Abe

Department of Orthopaedic Surgery, Graduate School of Medicine, Chiba University,

1-8-1 Inohana, Chuo-ku, Chiba 260-8670, Japan

Tel: +81-43-226-2117, Fax: +81-43-226-2116, E-mail: abeabeabe04@yahoo.co.jp
} 
of lumbar fusion increased by three-fold between 1992 and 2003 [2]. The Japanese Society for Spine Surgery and Related Research reported that the number of registered patients who underwent spinal surgery by their certified surgeons in 2011 had almost doubled that during the previous 10 years [3].

It has been reported that the conventional midline posterior approach for lumbar spine surgery causes significant muscle injury, which is particularly severe if powerful self-retaining retractors are used $[4,5]$. Gejo et al. [6] reported that muscle retraction for an extended time during the operation causes more severe back musclegdamage and a higher incidence of postoperative low back pain in human patients.

Regarding skin injury, Kajita et al. [7] reported that painful skin scar had induced hyperalgia in rats, which might cause plastic changes in the central nervous system. However, the relationship between muscle injury and postoperative pain has not been clarified. Therefore, the purpose of the current study was to determine the relationship between the histological variation of injured muscle and the production of calcitonin gene-related peptide (CGRP) in the dominant nerve during the first 3 weeks after experimental back muscle injury in rats.

\section{Materials and Methods}

All animal procedures and protocols were approved by the ethics committee of our university and followed the United States National Institute of Health Guidelines for the Care and Use of Laboratory Animals (1996 revision). We used 30 male, 8-week-old Sprague-Dawley rats. Each rat weighed approximately $250 \mathrm{~g}$ at the time of muscle injury. Before injury induction, the rats were anesthetized with ethyl ether. If a withdrawal reflex occurred, an additional anesthetic was administered until no response was noted. Muscle contusion was then induced without a skin incision by dropping a $115 \mathrm{~g}$ weight from a height of $1 \mathrm{~m}$ onto an impactor placed on the right medial paravertebral musculature (Fig. 1). Fluoro-Gold (FG; Fluorochrome, Denver, CO, USA), a retrograde neuronal tracer, was injected into both sides of the paravertebral muscle to label afferent sensory nerves.

\section{Histology}

The muscle injured site (i.e., the right side) and uninjured site (i.e., the left side) were dissected from the back muscle under anesthesia with sodium pentobarbital $(40 \mathrm{mg} / \mathrm{kg}$, intraperitoneally) at 1,2, and 3 weeks (12 rats, four rats per time period) after the muscle injury, and the sites were transcardially perfused with $0.9 \%$ saline, and subsequently with $500 \mathrm{~mL}$ of $4 \%$ paraformaldehyde in a phosphate buffer solution (PBS; 0.1 M, pH 7.4). Each formalin fixed tissue specimen was embedded in paraffin after dehydration for 14 hours using an ascending series of ethanol concentrations in Tissue-Tek VIP (M1500, Sakura Finetek Japan Co., Ltd., Tokyo, Japan). Subsequently, 4- $\mu$ m-thick sections were made from these paraffin blocks using a sliding microtome (LS113, Yamato Kohki Industrial Co., Ltd., Saitama, Japan), following which they were placed on glass slides (\#5116, Muto Pure Chemicals Co., Ltd., Tokyo, Japan). After deparaffinization with xylene and ethanol, these sections were stained with hematoxylin and eosin (H\&E). The sections were finally overlaid with the Entellan new mounting medium (Merck KGaA,

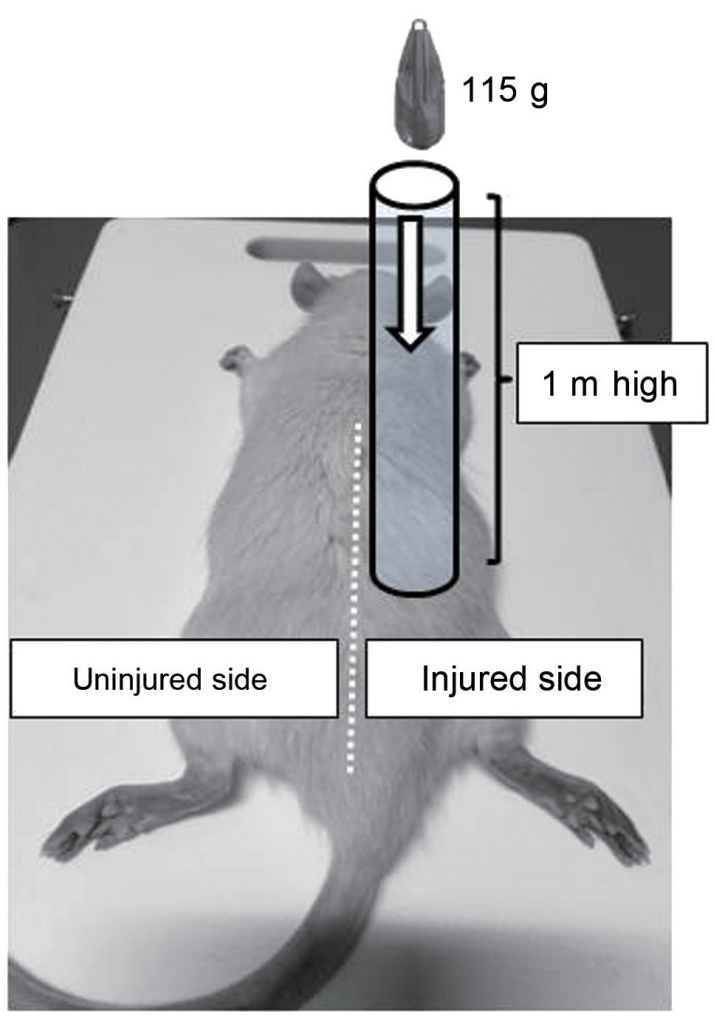

Fig. 1. We defined the right-side back muscle as the injured site and the left-side back muscle as the uninjured site. Under anesthesia, 8-week-old male Sprague-Dawley rats were injured on their right-side back muscle using a $115 \mathrm{~g}$ weight. The weight was dropped from a height of $1 \mathrm{~m}$ and was controlled by a pipe-shaped device. 
Darmstadt, Germany) after dehydration with a graded series of ethanol and xylene concentrations. A professional animal pathologist observed the slides under a microscope (BH20, Olympus Corp., Tokyo, Japan), and the degree of each finding was evaluated semiquantitatively. We evaluated the presence or absence of histological degeneration, bleeding, and neutrophil recruitment for each slice. We also compared the histological changes over time in the injured site against those in the uninjured site.

\section{Immunohistochemistry for CGRP}

The L2 dorsal root ganglia (DRG) of the injured and uninjured sides were removed at 1,2, and 3 weeks after the contusion injury. According to a previous report, the paravertebral musculature is under a significant degree of L2 control [8]. The proportion of FG-labeled neurons also showing immunoreactivity for CGRP, a marker of inflammatory pain, was then determined. Endogenous tissue peroxidase activity was quenched by soaking sections in $0.3 \%$ hydrogen peroxide solution in $0.01 \mathrm{M}$ PBS for 30 minutes. The specimens were then treated for 90 minutes at room temperature in a blocking solution consisting of $0.3 \%$ Triton X-100 and 3\% skim milk in 0.01 M PBS. The sections were labeled using a primary rabbit antibody to CGRP (Chemicon, Temecula, CA, USA) diluted to $1: 1,000$ in a blocking solution, and then incubated for 20 hours at $4^{\circ} \mathrm{C}$. To detect CGRP immunoreactivity in DRG, the sections were incubated with a goat anti-rabbit Alexa Fluor 488 fluorescent antibody conjugate (1:400, Molecular Probes, Eugene, OR, USA). The sections were examined under a fluorescence microscope, following which the number of FG-labeled, CGRP-immunoreactive (ir), and both FG-labeled and CGRP-ir neurons were determined in 10 randomly selected areas of each DRG section.

\section{Statistical analysis}

We used the $t$ test to compare the proportion of CGRPir FG-positive neurons in DRG at 1,2, and 3 weeks after muscle injury. $p<0.05$ was considered significant.

\section{Results}

\section{Histology}

Changes in the histology of the muscle samples over time at the injured site are shown in Fig. 2. H\&E staining of the paravertebral muscle showed infiltration of the inflammatory cells and the presence of granulation tissue in the injured part as well as neovascular hyperplasia on the ipsilateral side compared with the contralateral side 1 week after injury. Moreover, the absorption of degenerated cell tissues and muscle fiber repair were observed 2 weeks after injury. Muscle atrophy occurred 3 weeks after injury; however, the atrophy was repaired via spontaneous replacement of muscle cells or fibers.

\section{Immunohistochemistry for CGRP}

Results of the L2 DRG immunohistochemistry assay are shown in Fig. 3. The percentage of cells double-labeled with FG and CGRP in FG-positive cells of the primary muscle was significantly increased 1 (injured side 51.9\% vs. uninjured side $25.6 \%, p<0.05$ ), 2 (injured side $41.1 \%$ vs. uninjured side $21.3 \%, p<0.05$ ), and 3 weeks (injured side $40.5 \%$ vs. uninjured side $20.4 \%$, $p<0.05$ ) after injury.
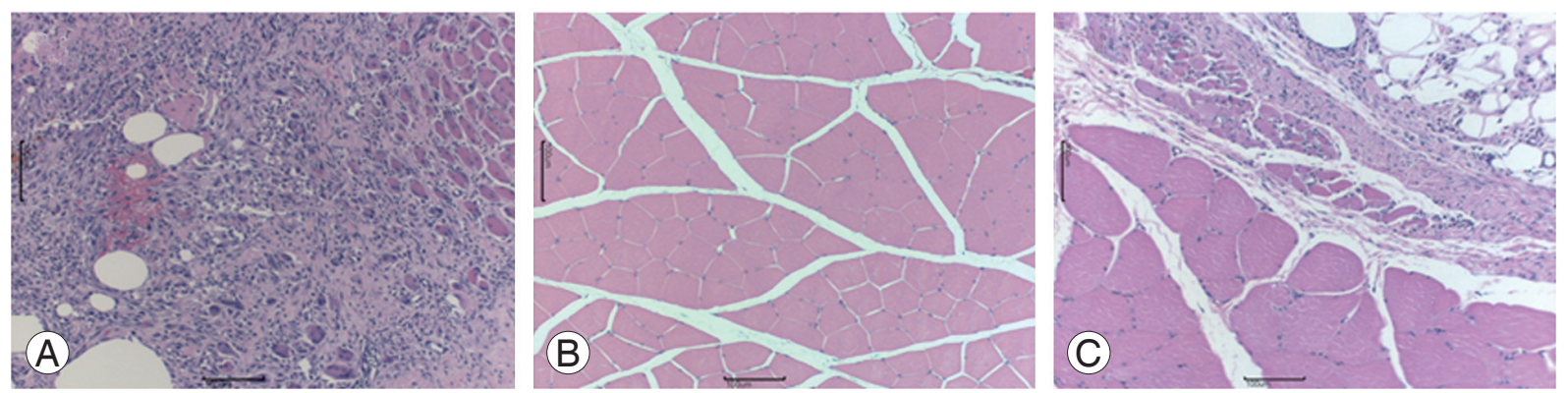

Fig. 2. Histological hematoxylin and eosin (H\&E) stained specimens of the muscle. (A) At 1 week after injury, infiltration of the inflammatory cells, granulation tissue, and vascularization were observed. (B) The absorption of degenerated cell tissues and repaired muscle fiber were observed 2 weeks after injury. (C) Muscle atrophy and the replacement of the muscle tissue were observed after 3 weeks. The scale bars are $100 \mu \mathrm{m}$. 


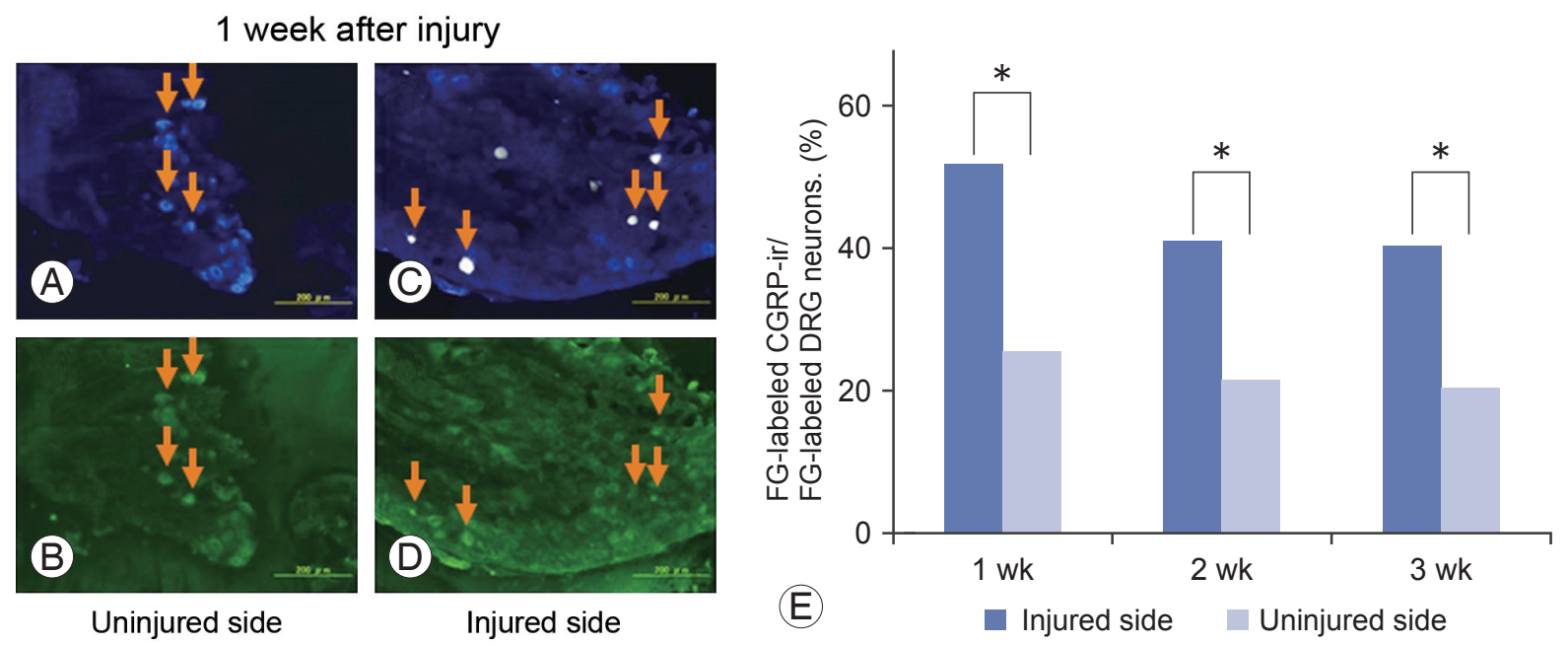

Fig. 3. Fluoro-Gold (FG)-labeled dorsal root ganglia (DRG) neurons (upper), and calcitonin gene-related peptide (CGRP)-immunoreactive (ir) DRG neurons of the same sections (lower). (A-D) DRG 1 week after injury. DRG from the injured side (C, D) versus those from the uninjured side (A, B). Red arrows indicate FG-labeled CGRP-ir DRG neurons. (E) The proportion of FG-labeled CGRP-immunoreactive doublelabeled neurons among all FG-labeled DRG neurons. ${ }^{*} p<0.05$.

\section{Discussion}

In the present study, inflammation at the injured site peaked at 1 week after injury, and scarring developed by the third week. Kawaguchi et al. [9] reported that regeneration began within 1 week and was accomplished within 6 weeks in a back muscle injury model in which a selfretractor was used for 3 hours in rats. However, in our drop mass model, the back muscle was damaged by only a single contusion; therefore, the injured tissue may have improved earlier.

In contrast, the pain neuropeptide in the DRG neurons innervating the injured muscle remained at an increased level 3 weeks after injury. In addition, in our previous study using an injured intervertebral disk model in rats, the inflammatory mediator at the injured site decreased to normal levels within 2 weeks; however, the CGRP-ir neurons among the DRG neurons innervating the responsible lesion remained at an increased level for at least 8 weeks [10].

Previously, it has been reported that a decrease in the trunk muscle strength is the main factor for chronic muscle pain [6]. We cannot deny that atrophy of the muscle tissue observed at 3 weeks could initiate postoperative muscle pain. However, our hypothetical inference was that the prolonged sensitization of the neuropeptide in DRG may produce postoperative back muscle pain. Alternatively, scarring in the damaged muscle may be responsible for a certain type of nociceptive signal being sent to DRG at
3 weeks after injury, regardless of the histological completion of local inflammation. Determining the causes for postoperative back muscle pain would require long-term research of the neuropeptide in DRG innervating the injured sites.

Regardless, our results indicate that the amount of muscle damage should be minimized during surgery. A study that used a self-retaining retractor in rats reported that 5 min of retraction release every 40 or 60 minutes could prevent severe muscle injury during posterior lumbar spine surgery [11]. In addition, minimally invasive spine surgery techniques have been reviewed as procedures to decrease muscle crush injuries [5]. The present study confirms the aforementioned points.

A limitation of our model was that the injury was caused by direct contusion; however, the power of traction and compression causes muscle damage during actual surgery. An intraoperative back muscle injury model should be constructed using retracting devices, such as those used by Kawaguchi et al. [9] in their muscle injury animal model. In addition, we did not quantify inflammation of the injured tissue through a histological method or immunohistochemistry technique. Moreover, a behavioral study should be performed to evaluate muscle pain, as performed by Miyagi et al. [12] and Sakuma et al. [13] using the CatWalk system in which an animal's gait can be analyzed. We are planning to resolve these issues through future research. 


\section{Conclusions}

In the rat model of the current study, inflammation at the injured site peaked at 1 week and was terminated by 3 weeks after injury. However, the sensory nervous system of DRG remained activated at 3 weeks. These results suggest that sensitization of the dominant nerve in DRG can protract injured muscle pain. This information may contribute in elucidating the underlying mechanism of residual postoperative back muscle pain.

\section{Conflict of Interest}

No potential conflict of interest relevant to this article was reported.

\section{Acknowledgments}

We thank laboratory technician Ikuko Tajiri for her expert advices and assistance.

\section{References}

1. Kim P, Kurokawa R, Itoki K. Technical advancements and utilization of spine surgery: international disparities in trend-dynamics between Japan, Korea, and the USA. Neurol Med Chir (Tokyo) 2010;50:8538.

2. Weinstein JN, Lurie JD, Olson PR, Bronner KK, Fisher ES. United States' trends and regional variations in lumbar spine surgery: 1992-2003. Spine (Phila Pa 1976) 2006;31:2707-14.

3. Imajo Y, Taguchi T, Yone K, et al. Japanese 2011 nationwide survey on complications from spine surgery. J Orthop Sci 2015;20:38-54.

4. Gejo R, Kawaguchi Y, Kondoh T, et al. Magnetic resonance imaging and histologic evidence of postoperative back muscle injury in rats. Spine (Phila Pa 1976) 2000;25:941-6.
5. Kim CW. Scientific basis of minimally invasive spine surgery: prevention of multifidus muscle injury during posterior lumbar surgery. Spine (Phila Pa 1976) 2010;35:S281-6.

6. Gejo R, Matsui H, Kawaguchi Y, Ishihara H, Tsuji $H$. Serial changes in trunk muscle performance after posterior lumbar surgery. Spine (Phila Pa 1976) 1999;24:1023-8.

7. Kajita Y, Suetomi K, Okada T, et al. Behavioral and neuropathological changes in animal models of chronic painful scar. J Orthop Sci 2013;18:1005-11.

8. Wakai K, Ohtori S, Yamashita M, et al. Primary sensory neurons with dichotomizing axons projecting to the facet joint and the low back muscle in rats. J Orthop Sci 2010;15:402-6.

9. Kawaguchi Y, Matsui H, Tsuji H. Back muscle injury after posterior lumbar spine surgery. Part 1: Histologic and histochemical analyses in rats. Spine (Phila Pa 1976) 1994;19:2590-7.

10. Miyagi M, Ishikawa $T$, Orita $S$, et al. Disk injury in rats produces persistent increases in pain-related neuropeptides in dorsal root ganglia and spinal cord glia but only transient increases in inflammatory mediators: pathomechanism of chronic diskogenic low back pain. Spine (Phila Pa 1976) 2011;36:2260-6.

11. Kawaguchi Y, Matsui H, Gejo R, Tsuji H. Preventive measures of back muscle injury after posterior lumbar spine surgery in rats. Spine (Phila Pa 1976) 1998;23:2282-7.

12. Miyagi M, Ishikawa $\mathrm{T}$, Kamoda $\mathrm{H}$, et al. Assessment of pain behavior in a rat model of intervertebral disc injury using the CatWalk gait analysis system. Spine (Phila Pa 1976) 2013;38:1459-65.

13. Sakuma Y, Miyagi M, Inoue G, et al. Muscle injury in rats induces upregulation of inflammatory cytokines in injured muscle and calcitonin gene-related peptide in dorsal root ganglia innervating the injured muscle. Muscle Nerve 2016;54:776-82. 